\title{
D-branes in a marginally deformed WZW model
}

\author{
Stefan Förste \\ Physikalisches Institut der Universität Bonn \\ Nussallee 12, 53115 Bonn, Germany
}

\begin{abstract}
In this talk we discuss symmetry preserving D-branes on a line of a marginally deformed $S U(2)$ WZW model. A semiclassical and a quantum theoretical approach are presented.
\end{abstract}

This talk is based on the publications [1, 2]. I thank Daniel Roggenkamp for a very enjoyable collaboration on [2].

\section{Outline}

After giving a short introduction and motivation I will present the semiclassical description of D-branes in the marginally deformed $S U(2)$ model. The second part of the talk addresses the quantum theoretical description of the D-branes reporting results which will be derived in more detail in[2]. I will finish with some concluding remarks.

\section{Introduction and Motivation}

Let us first discuss the exact marginal deformations in the case of closed strings. We know that a (perturbative) string vacuum corresponds to a two dimensional conformal field theory $(\mathrm{CFT})$. An operator $\mathcal{O}$ is called marginal if it has conformal dimension $(1,1)$. A typical example of a marginal operator is the product of a chirally conserved current times an anti-chirally conserved current. An infinitesimal perturbation of the action by a worldsheet integral over a marginal operator preserves conformal invariance. Under certain circumstances [3] the infinitesimal perturbation can be integrated up to a finite exact marginal deformation. This results in a one parameter family of CFTs, where two adjacent points on that line are connected by an infinitesimal marginal perturbation. Hence, exact marginal deformations yield a line in the space of string vacua and thus help to gain insight into the structure of the moduli space of string theories. The inclusion of D-branes into this picture provides information about the moduli space of string theories in the presence of D-branes. The inclusion of D-branes means that we investigate CFTs on a worldsheet with boundaries. Such a theory could also be perturbed by marginal boundary operators. Deformations resulting from such a perturbation will not be discussed in the present talk. 
Our plan is to consider as an example the $S U(2)$ WZW model and deformations thereof. The sigma model description of this class of CFTs is discussed e.g. in [4, 5, 6].

\section{Semiclassical Description}

Before going to the deformed case let us recall the picture for symmetry preserving D-branes on $S U(2)$. Two useful parameterizations of an $S U(2)$ group element are

$$
\begin{aligned}
g & =\cos \chi+i \sin \chi \cos \vartheta \sigma^{1}+i \sin \chi \sin \vartheta \cos \varphi \sigma^{2}+i \sin \chi \sin \vartheta \sin \varphi \sigma^{3} \\
& =\cos x \cos \tilde{\theta}-i \sin x \sin \theta \sigma^{1}+i \sin x \cos \theta \sigma^{2}+i \cos x \sin \tilde{\theta} \sigma^{3},
\end{aligned}
$$

with the parameter ranges $\chi=0 \ldots \pi, \vartheta=0 \ldots \pi, \varphi=0 \ldots \pi$ and $x=0 \ldots \pi / 2, \theta=$ $-\pi \ldots \pi, \tilde{\theta}=-\pi \ldots \pi$. The first parameterization is useful for specifying the location of the D-branes whereas in the second one the marginal deformations look simple. The corresponding target space metric is the Cartan-Killing metric

$$
\begin{aligned}
d s^{2} & =k \alpha^{\prime}\left\{d \chi^{2}+\sin ^{2} \chi\left(d \vartheta^{2}+\sin ^{2} \vartheta d \varphi^{2}\right)\right\} \\
& =k \alpha^{\prime}\left\{d x^{2}+\sin ^{2} x d \theta^{2}+\cos ^{2} x d \tilde{\theta}^{2}\right\}
\end{aligned}
$$

leading to an $S^{3}$ geometry. The radius of this three sphere is quantized, $k \in \mathbb{N}[\sqrt{7}$.

The symmetry preserving D-branes were described in $8 \|$ as conjugacy classes of a fixed group element. These are spherical branes breaking the $S U(2) \times S U(2)$ Kac-Moody to a diagonal $S U(2)$ symmetry. In terms of isometries the $S O(4)$ of $S^{3}$ is broken to an $S O(3)$ acting as rotations along the branes. Further, a topological argument let the authors of 8 to a quantization condition on the position of the D-branes. In the parameterization (11) the position of the brane is specified by fixing the value of $\chi$ and the quantization condition reads

$$
\chi \in \frac{\pi \mathbb{Z}}{k} .
$$

Later in [10] the position of the D-branes was studied by probing the geometry with gravitons and it was found that (3) is quantum corrected to

$$
\chi \in \frac{\pi(2 j+1)}{k+2}, j=0, \frac{1}{2}, 1, \ldots, \frac{k}{2} .
$$

We will come back to this method of investigating the D-brane geometry later. In the present section we consider the large $k$ limit in which the quantization conditions coincide apart from the zero dimensional branes at the poles. We will not include branes at the poles into the discussion.

At first sight this picture seems counterintuitive since the two-dimensional tension-full branes wrapping contractable cycles are expected to shrink to zero volume. The mechanism responsible for stabilizing the two dimensional branes has been given in 11, 12] (see also 13. for more general groups): The quantization condition on the position of the D-brane (3) corresponds to a quantization condition on the F-flux through the D-brane.

\footnotetext{
${ }^{1} \mathrm{~A}$ semiclassical discussion of symmetry breaking branes can be found in 9 .
} 
The metric for the deformed $S U(2)$ model can be found in e.g. [5]. In the coordinates (2) it reads

$$
d s^{2} / k \alpha^{\prime}=d x^{2}+\frac{\sin ^{2} x d \theta^{2}+R^{2} \cos ^{2} x d \tilde{\theta}^{2}}{\cos ^{2} x+R^{2} \sin ^{2} x},
$$

where the deformation parameter $R$ takes values in $(0, \infty)$. The value $R=1$ corresponds to the undeformed model. In the limit $R \rightarrow \infty(R \rightarrow 0)$ the $\theta(\tilde{\theta})$ coordinates decouple and the limiting geometries are $S U(2) / U(1)$ times a free boson on a circle of vanishing radius (which can be T-dualized to the real line). The geometry of $S U(2) / U(1)$ can be conformaly compactified to a disk [14]. Away from $R=1$ the $S U(2) \times S U(2)$ Kac-Moody symmetry is broken to a $U(1) \times U(1)$ symmetry. The corresponding chirally and anti-chirally conserved currents combine to the marginal operator taking the model from a point $R$ to an infinitesimal close point $R+\delta R$. The fate of the symmetry preserving D-branes was studied in 11 where the following rules were imposed. The D-branes should break the $U(1) \times U(1)$ to a residual $U(1)$ at generic $R$ and for $R=1$ they should be identical to the previously discussed symmetry preserving branes on $S U(2)$. Further an F-flux quantization condition should be satisfied. This lead to the result that the position of the D-branes expressed in the coordinates (2) does not change with $R$. In the remaining part of the talk we want to confirm this result by a quantum theoretical discussion.

\section{Quantum Theoretical Description}

In order to go beyond the semiclassical treatment of the previous section we should construct the boundary states for the symmetry preserving D-branes on the line of deformed models. What we will do is to give a set of boundary states on the family of deformed models and claim that a certain subset of those boundary states corresponds to the symmetry preserving D-branes. Afterwards we will justify our claim by showing that in the large $k$ limit the geometries are identical. In order to construct the boundary states on the deformed models an alternative description of the deformation turns out to be useful. This description was proposed in 15. The statement is that for arbitrary $R$ there is an identification

$$
\text { Deformed Model }=\left(\mathrm{pf}_{k} \times u(1)_{\sqrt{k} R}\right) / \mathbb{Z}_{k}
$$

Let us briefly sketch the ingredients of this construction. First the parafermions $\left(\mathrm{pf}_{k}\right)$ posses a $\mathbb{Z}_{k} \times \mathbb{Z}_{k}$ symmetry with the currents

$$
\psi_{l}(z) \text { and } \psi_{l}^{\dagger}(z)
$$

whose charges are $(l, 0)$ and $(-l, 0)$, respectively $(l=0, \ldots, k-1)$. One example for an OPE in $\mathrm{pf}_{k}$ is

$$
\psi_{l}(z) \psi_{p}(0) \sim z^{-l p / k}\left(\psi_{l+p}(0)+\mathcal{O}(z)\right) .
$$

Since moving $\psi_{l}$ once around $\psi_{p}$ yields a phase which is not necessarily \pm 1 the model is called parafermion model.

The other ingredient is a free compact boson

$$
\phi(z, \bar{z})=\phi(z)+\bar{\phi}(\bar{z}),\langle\phi(z) \phi(0)\rangle=-2 \log z .
$$


That the above model is for $R=1$ equivalent to the $S U(2)$ model has been pointed out in 16]. The $S U(2)$ currents can be represented as follows,

$$
\begin{aligned}
J^{+}(z) & =\sqrt{k} \psi_{1}(z): e^{\frac{i \phi(z)}{\sqrt{k}}}: \\
J^{-}(z) & =\sqrt{k} \psi_{1}^{\dagger}(z): e^{\frac{-i \phi(z)}{\sqrt{k}}}: \\
J^{3}(z) & =\sqrt{k} \partial_{z} \phi(z),
\end{aligned}
$$

which can be checked by comparing the OPE's. The right hand sides are invariant under a $\mathbb{Z}_{k}$ transformation acting on the parafermions and simultaneously shifting the free boson. Thus the $\mathbb{Z}_{k}$ orbifold group in (6) is a diagonal subgroup of the $\mathbb{Z}_{k} \times \mathbb{Z}_{k}$ symmetry in pf combined with a discrete shift along the $u(1)$. The deformed models are now reached by changing the size of the circle on which the boson $\phi$ lives or more generally spoken deforming the Cartan torus of the group. This can be confirmed by matching the zero mode spectrum of the orbifold (including twisted sectors) with the eigenvalues of the Laplacian (with non-trivial dilaton, see e.g.[14]) appearing in the effective closed string action.

The construction of boundary states in the description (6) turns out to be rather simple since all the input one needs can be found in the literature. The boundary states in $\mathrm{pf}_{k}$ have been constructed in $[14]^{2}$ and the boundary states for a free boson on a circle are simple. A boundary state on the orbifold is obtained by tensoring these two and adding all $\mathbb{Z}_{k}$ images. Our claim is that the symmetry preserving D-branes of the previous section arise by combining an A-type boundary state of $\mathrm{pf}_{k}$ with Dirichlet-type boundary state of the free boson (with a certain position[). The A-type boundary state of the parafermion theory reads

$$
\left.\left|B_{(j, n)}^{k}\right\rangle=\sum_{\left(j^{\prime}, n^{\prime}\right)} \frac{S_{(j, n),\left(j^{\prime}, n^{\prime}\right)}^{k}}{\sqrt{S_{(0,0)\left(j^{\prime}, n^{\prime}\right)}^{k}}}\left|\left(j^{\prime}, n^{\prime}\right)\right\rangle\right\rangle,
$$

where the notation follows 14. In particular the pair $(j, n)$ labels the highest weight states and the $S$ matrix arises in modular transformations of the closed string partition function. The action of an $l$ th order element $\ell$ of the orbifold group $\mathbb{Z}_{k}$ follows from its action on the Ishibashi state 25

$$
\left.\left.\ell\left|\left(j^{\prime}, n^{\prime}\right)\right\rangle\right\rangle=\exp \left\{2 \pi i l n^{\prime} / k\right\}\left|\left(j^{\prime}, n^{\prime}\right)\right\rangle\right\rangle .
$$

The Dirichlet-type boundary state on $u(1)_{\sqrt{k} R}$ reads

$$
\left.\left|D^{\sqrt{k} R}(x)\right\rangle=\sum_{p \in \mathbb{Z}} \frac{e^{2 \pi i p x / k}}{\sqrt{\sqrt{k} R}}|(p, 0)\rangle\right\rangle_{D},
$$

where the momentum number $p$ labels the highest weight states and $x$ specifies the position of the D-brane. We see that $R$ enters only as a normalization. The $\mathbb{Z}_{k}$ action on the Ishibashi state in (10) is

$$
\left.\ell|(p, 0)\rangle\rangle_{D}=\exp \{-2 \pi i l p / k\}|(p, 0)\rangle\right\rangle_{D} .
$$

The sum over the $\mathbb{Z}_{k}$ images of the tensor product is non-vanishing only if the momentum number of the Dirichlet Ishibashi state coincides with $n^{\prime}$ in the $\mathrm{pf}_{k}$ Ishibashi state which is

\footnotetext{
${ }^{2}$ For discussions of D-branes on cosets see also $17,18,19,20,21,22,23$.

${ }^{3}$ For general positions $x$ the D-brane is localized on a twisted conjugacy class 24 .
} 
labeled by the pair $\left(j^{\prime}, n^{\prime}\right)$ as in[14] (see also (8)). For a certain value for the position of the Dirichlet boundary state the boundary state on the orbifold takes the form of an $S U(2)$ Cardy state 26] belonging to symmetry preserving branes on $S U(2)$.

It remains to show that these boundary states are indeed the ones we found in the semiclassical considerations, before. To this end, we need to derive the geometry of the D-brane given by the boundary state. The problem of deriving the geometry from a given boundary state has been analyzed in 27 for flat target spaces and in 10 for group manifolds. In both cases one identifies the D-brane geometry as the set of points on which the overlap between a closed string graviton state and the boundary state does not vanish (for $k \rightarrow$ $\infty)$. For our purpose the discussion in 10 can be used in a straightforward way. As we already mentioned the boundary state in the deformed model takes the form of a symmetry preserving $S U(2)$ Cardy state. The other information one needs is that the form of the eigenfunctions of

$$
\frac{e^{2 \Phi}}{\sqrt{G}} \partial_{\mu}\left(e^{-2 \Phi} \sqrt{G} G^{\mu \nu} \partial_{\nu}\right)
$$

does not change with $R$ (here $\Phi$ and $G$ denote the dilaton and metric in the deformed model). This implies that the highest weight contribution to the closed string graviton state does not change its form under the deformation. Using this and going through the discussion of 10 one finds that the D-branes are localized on conjugacy classes of a fixed group element. This means that the D-brane position expressed in the coordinates of the undeformed model does not change under the deformation.

\section{Conclusions and Outlook}

In the present talk we have given the semiclassical and the algebraic description for symmetry preserving D-branes along the line of exact marginal deformations of the $S U(2)$ WZW model. The presentation has been rather short, more details on the semiclassical description can be found in [1], whereas the algebraic approach will be discussed in [2]. Ref. [2] will contain more results than presented here including symmetry breaking branes and generalizations beyond the $S U(2)$ example. More examples for interesting questions in the present context concern: Flows under relevant perturbations (see e.g. 28, 29]), the inclusion of marginal boundary perturbations, extension to non-compact groups (see e.g. 30, 31]) and many others.

\section{Acknowledgement}

I thank the organizers of the conference for the kind invitation to present this talk and for creating a very pleasant and stimulating atmosphere.

This work is supported in part by the European Community's Human Potential Programme under contracts HPRN-CT-2000-00131 Quantum Spacetime, HPRN-CT-2000-00148 Physics Across the Present Energy Frontier and HPRN-CT-2000-00152 Supersymmetry and the Early Universe, and INTAS 00-561.

\section{References}

[1] S. Förste, JHEP 0202 (2002) 022 arXiv:hep-th/0112193.

[2] S. Förste and D. Roggenkamp, to appear. 
[3] S. Chaudhuri and J. A. Schwartz, Phys. Lett. B 219 (1989) 291.

[4] S. F. Hassan and A. Sen, Nucl. Phys. B 405 (1993) 143 arXiv:hep-th/9210121.

[5] A. Giveon and E. Kiritsis, Nucl. Phys. B 411 (1994) 487 arXiv:hep-th/9303016.

[6] K. Sfetsos and A. A. Tseytlin, Phys. Rev. D 49 (1994) 2933 arXiv:hep-th/9310159].

[7] E. Witten, Commun. Math. Phys. 92 (1984) 455.

[8] A. Y. Alekseev and V. Schomerus, Phys. Rev. D 60 (1999) 061901 arXiv:hepth/9812193.

[9] K. Behrndt, Nucl. Phys. B 414 (1994) 114 arXiv:hep-th/9304096.

[10] G. Felder, J. Fröhlich, J. Fuchs and C. Schweigert, J. Geom. Phys. 34 (2000) 162 arXiv:hep-th/9909030.

[11] C. Bachas, M. R. Douglas and C. Schweigert, JHEP 0005 (2000) 048 arXiv:hepth/0003037.

[12] J. Pawelczyk, JHEP 0008 (2000) 006 [arXiv:hep-th/0003057.

[13] P. Bordalo, S. Ribault and C. Schweigert, JHEP 0110 (2001) 036 arXiv:hepth/0108201].

[14] J. M. Maldacena, G. W. Moore and N. Seiberg, JHEP 0107 (2001) 046 arXiv:hepth/0105038.

[15] S. K. Yang, Phys. Lett. B 209 (1988) 242.

[16] D. Gepner and Z. a. Qiu, Nucl. Phys. B 285 (1987) 423.

[17] K. Gawedzki, Annales Henri Poincare 3 (2002) 847 arXiv:hep-th/0108044.

[18] S. Parvizi, Phys. Lett. B 520 (2001) 367 arXiv:hep-th/0108095.

[19] S. Elitzur and G. Sarkissian, Nucl. Phys. B 625 (2002) 166 arXiv:hep-th/0108142.

[20] S. Fredenhagen and V. Schomerus, arXiv:hep-th/0205011.

[21] H. Ishikawa, Nucl. Phys. B 629 (2002) 209 arXiv:hep-th/0111230.

[22] T. Kubota, J. Rasmussen, M. A. Walton and J. G. Zhou, Phys. Lett. B 544 (2002) 192 arXiv:hep-th/0112078.

[23] M. A. Walton and J. G. Zhou, arXiv:hep-th/0205161.

[24] S. Stanciu, JHEP 0001 (2000) 025 arXiv:hep-th/9909163.

[25] N. Ishibashi, Mod. Phys. Lett. A 4 (1989) 251.

[26] J. L. Cardy, Nucl. Phys. B 324 (1989) 581.

[27] P. Di Vecchia, M. Frau, I. Pesando, S. Sciuto, A. Lerda and R. Russo, Nucl. Phys. B 507 (1997) 259 arXiv:hep-th/9707068. 
[28] A. Recknagel, D. Roggenkamp and V. Schomerus, Nucl. Phys. B 588 (2000) 552 arXiv:hep-th/0003110.

[29] S. Fredenhagen and V. Schomerus, arXiv:hep-th/0104043.

[30] S. Förste, Phys. Lett. B 338 (1994) 36 arXiv:hep-th/9407198.

[31] R. Manvelyan, arXiv:hep-th/0206218. 